\title{
A Hierarchical Approach to Optimize LNG Fractionation Units
}

\author{
Hassan E. Alfadala ${ }^{a^{*}}$, Bilal M. Ahmad ${ }^{a}$, Abdulla F. Warsame ${ }^{b}$ \\ ${ }^{\text {a }}$ Department of Chemical Engineering, University of Qatar \\ P. O. Box 2713, Doha Qatar \\ ${ }^{\mathrm{b}}$ Qatar Liquefied Gas Company \\ P. O. Box 22666, Doha Qatar
}

\begin{abstract}
The objective of this paper is to optimize the thermal performance of a fractionation unit within a liquefied natural gas (LNG) facility. Typical fractionation units in an LNG facility consisting of three distillation columns, namely de-ethanizer, de-propanizer and de-butanizer were used in this study. A hierarchical approach is developed to optimizing the system. In this approach, increasing levels of model complexity are used and various thermal targets are set and implemented. The column targeting tool available within the simulation package of Aspen Plus ${ }^{\odot}$ software was used to optimize a fractionation unit in an LNG facility. First, integrated thermal analysis was used in identifying design targets for improvements in energy consumption and efficiency. The column targeting tool is used in the design of distillation columns by setting targets to reduce utility cost, improve energy efficiency, reduce capital investment and facilitate column debottlenecking. Starting from a short-cut distillation design calculation using the DSTWU method which is based on the well-known Fenske-Underwood-Gilliland correlations, the minimum and actual reflux ratios, minimum and actual number of stages, optimum feed location and condenser and reboiler duties were estimated. These estimates were used as starting points in the rigorous fractionation column design method RADFRAC available in Aspen Plus ${ }^{\odot}$. The column Grand Composite Curve (CGCC) for each column was generated to give an insight of the actual operation and guide the optimization process. Starting with appropriate feed placement, the CGCC will show the scope for reflux ratio modification by increasing the number of stages. Feed would be either preheated or precooled due to the availability of sharp enthalpy change in the condenser or reboiler side. Finally, the scope for a side condensing or side reboiling can be identified from the area beneath or above the CGCC pinch point.
\end{abstract}

\section{Introduction}

Today in the chemical process industry, distillation is considered to be one of the most used unit operation. Such operation consumes the highest amount of energy compared to the rest of the unit operations in any facility. This is due to the fact that distillation is based on boiling temperature difference between the light key and the heavy key. Thus

\footnotetext{
*Author to who correspondence should be addressed, e-mail: alfadala@qu.edu.qa
} 
any attempt to investigate and understand the energy flow in distillation columns would benefit the overall process economy and reduce product cost. In particular, distillation units are core elements in an LNG facility.

About a decade ago Dhole and Linnhoff, 1993, had introduced the concept of Column Grand Composite Curve (CGCC). Since then, this concept has been extended (e.g., Gadalla et al., 2003). The CGCC is based on the Practical Near-Minimum Thermodynamic Condition (PNMTC) approximation. These curves could be generated using Aspen Plus Column Targeting tool, Aspen Technology Inc., 2003. This work employs Aspen Plus simulation package capabilities to optimize LNG fractionation unit.

\section{Overall optimization approach}

In order to strike a proper balance between computational efficiency and result accuracy, we develop a hierarchical approach as shown in Figure 1. Starting with the short-cut column model DSTWU available within Aspen Plus model library, which is based on the well-known Fenske-Underwood-Gilliland correlations, Seader and Henly, 1998, the minimum and actual reflux ratios, minimum and actual number of stages, feed location, condenser cooling requirement and reboiler heating requirement were estimated.

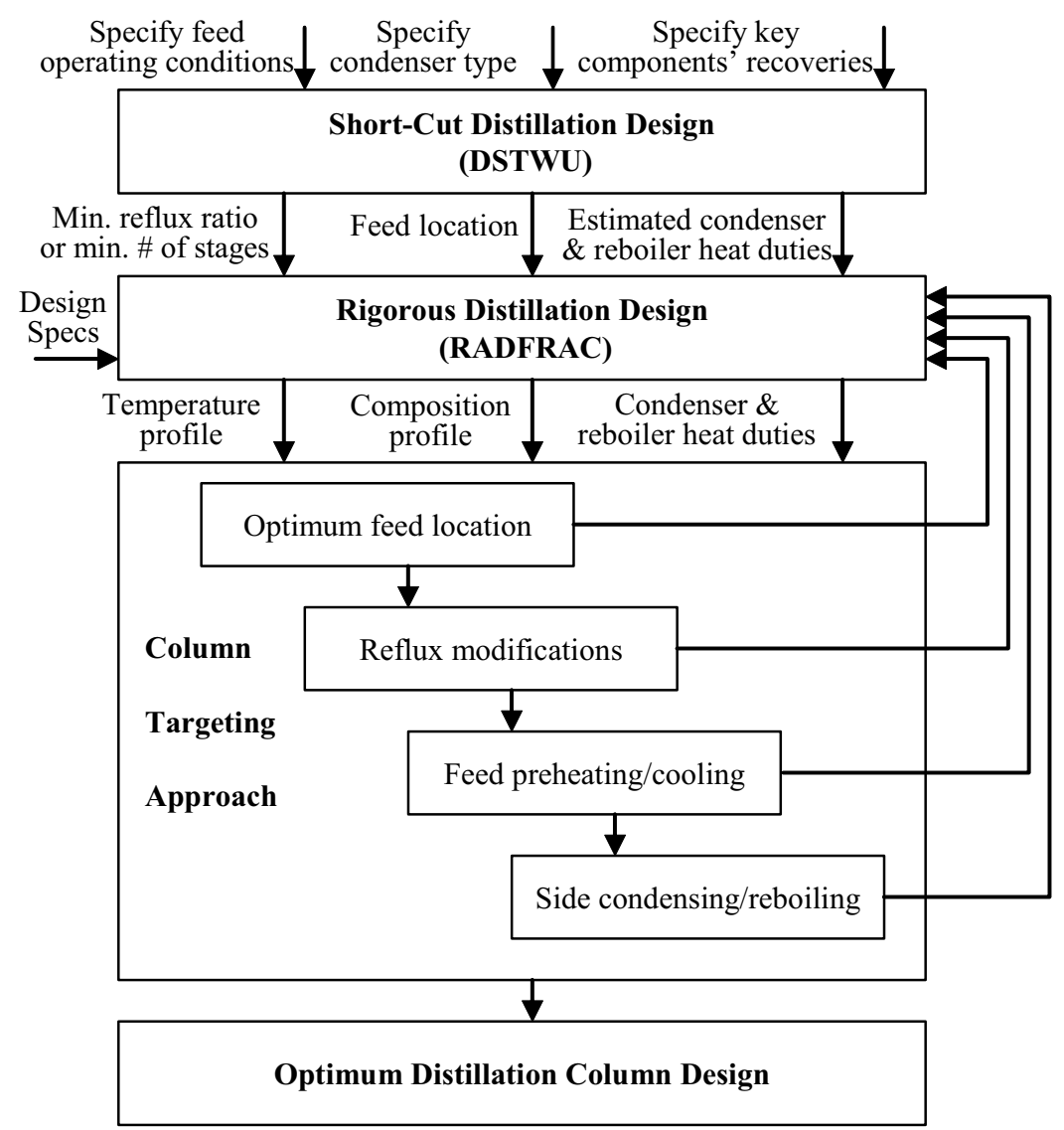

Figure 1. Overall optimization approach 
In order to use the DSTWU model, feed operating conditions, condenser type, key components and their recoveries must be specified.

Outputs from the short-cut model will include the minimum reflux ratio, minimum number of stages, feed stage location, and the estimated condenser and reboiler heat duties.

All of the above data is required to start the rigorous RADFRAC model which is also available within Aspen Plus model library. The user should specify the required design specifications at this stage. As an example of a design specification would be the targeted purity of the light key in the top product. For each design specification, there must be a variable which will have an upper bound and a lower bound. The condenser duty could be used as an example for the vary type in the RADFRAC model. In order to demonstrate the advantages of the column targeting tool, the following case study has been worked out as an example.

\section{Case study}

An LNG facility fractionation unit was optimized as a case study using the above mentioned overall optimization approach. The specifications of the feed and products for the case study are given in Table 1. These are representative data of a typical LNG fractionation unit.

Table 1. Feed and product specifications for the case study

\begin{tabular}{llll}
\hline Properties & Feed & Top product & Bottom product \\
Mass Flow rate $(\mathrm{kg} / \mathrm{hr})$ & 25736.0 & 13476.4 & 12259.6 \\
Temperature $\left({ }^{\circ} \mathrm{C}\right)$ & 77.4 & 48.3 & 124.2 \\
Pressure $($ bar $)$ & 6.62 & 6.52 & 6.76 \\
Vapor fraction & 0.38 & 0.00 & 0.00 \\
Mole fractions & & & \\
Propane & 0.05388 & 0.13260 & 0.00000 \\
i-Butane & 0.14808 & 0.27648 & 0.00002 \\
n-Butane & 0.29493 & 0.55049 & 0.00033 \\
Methyl-Butane & 0.13301 & 0.03696 & 0.26470 \\
n-Pentane & 0.12368 & 0.00347 & 0.29627 \\
n-Heptane & 0.23114 & 0.00000 & 0.40624 \\
Benzene & 0.00941 & 0.00000 & 0.02122 \\
Toluene & 0.00587 & 0.00000 & 0.01122 \\
\hline
\end{tabular}

As mentioned above, in the overall optimization approach, as a starting point a converged simulation for the distillation column using the DSTWU must be obtained first. Thereafter, such outputs are fed into the rigorous RADFRAC model in order to construct the column grand composite curve as shown in Figure 2. The following are the four steps for column targeting approach.

\section{Feed Location}

Inspecting the CGCC shown in Figure 2 the feed is appropriately located due to no distortions in the CGCC. If the feed misplaced otherwise, the CGCC would be shifted towards either the condenser or the reboiler side. 


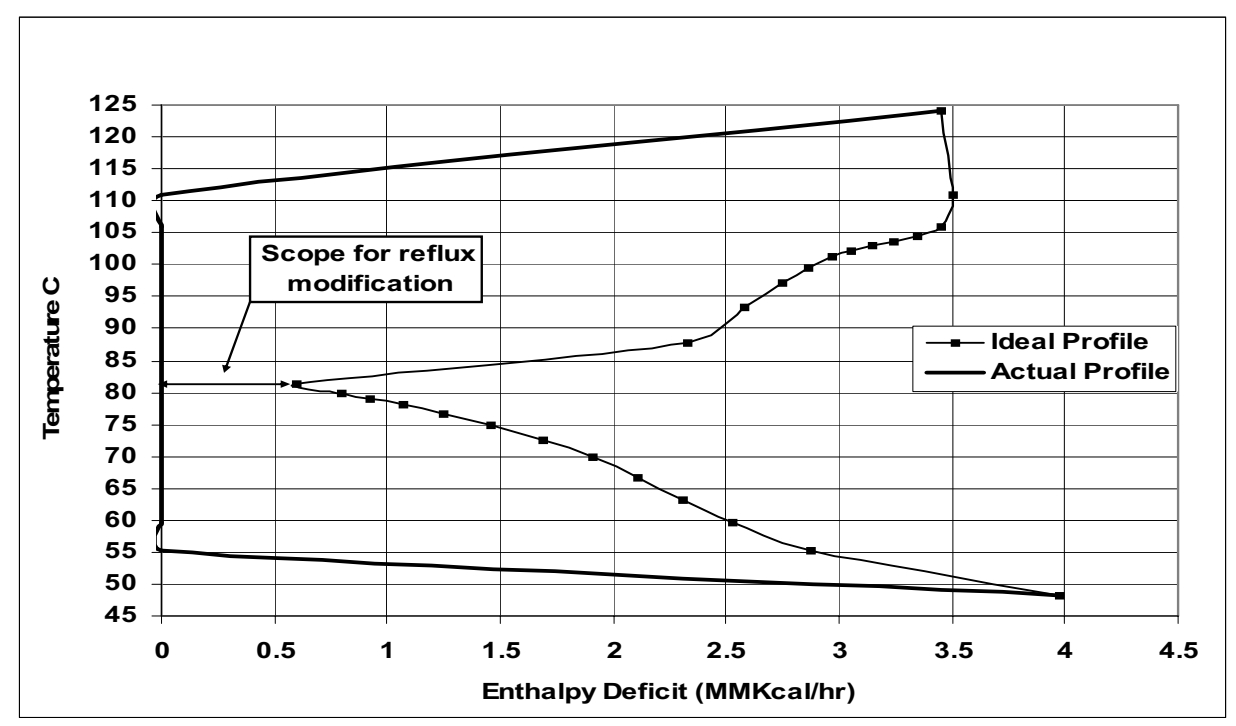

Figure 2. Debutanizer Column Grand Composite Curve $(T-H)$

\section{Reflux ratio adjustment}

The horizontal distance in Figure 2 between the y-axis and the pinch point shows the scope for reflux ratio modification. Of course, this is a trade-off between capital cost and operating expenditure, which is due to the fact that whenever the reflux ratio is changed the operating cost gets affected, thus changing the number of stages of the distillation column, which accordingly will have an impact on the capital cost.

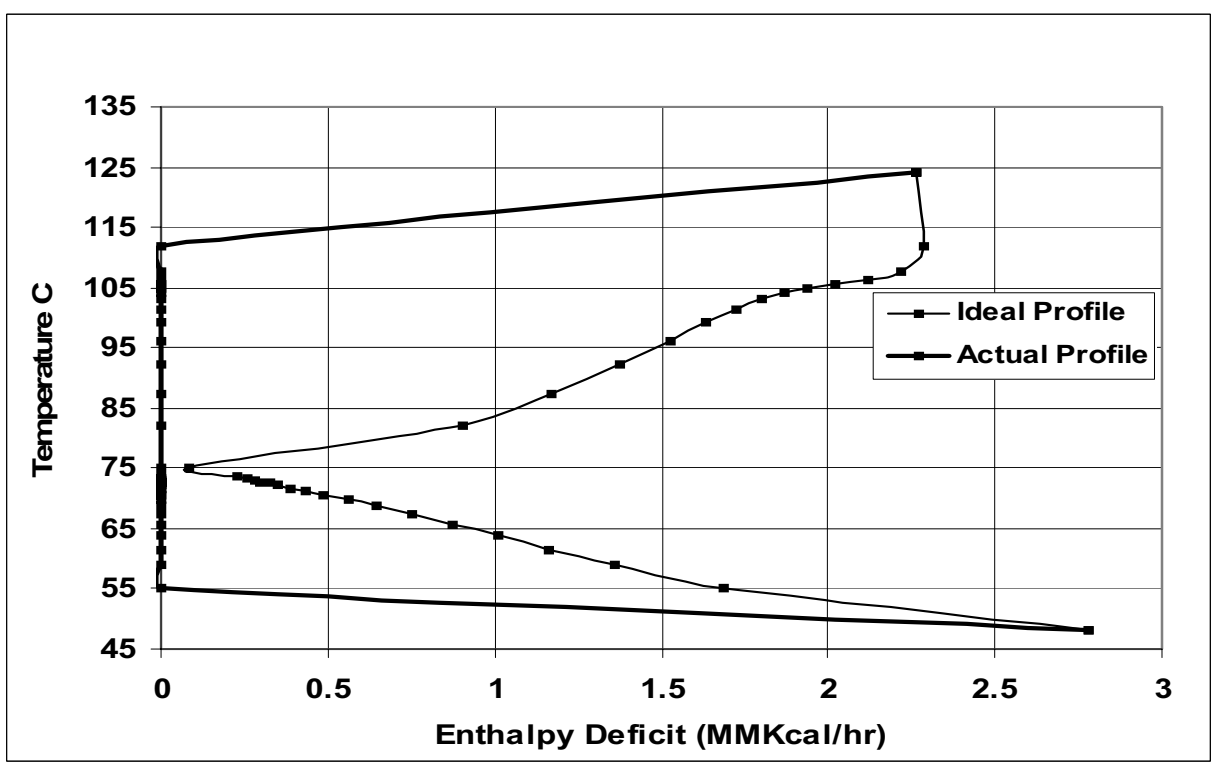

Figure 3. Debutanizer Column Grand Composite Curve after reflux modification 
Figure 3 shows the CGCC after the reflux ratio has been reduced to minimize the distance between the pinch point and the y-axis. In order to meet the same design specifications, the number of stages had increased in this case from 25 to 33 stages.

\section{Feed conditioning}

Sharp enthalpy changes on the CGCC will determine the need for pre-heating or precooling the feed. Figure 3 shows that there is a sharp enthalpy change on the condenser side, which determines the requirement for a pre-cooler. The resulting CGCC after the pre-cooling effects is shown in Figure 4.

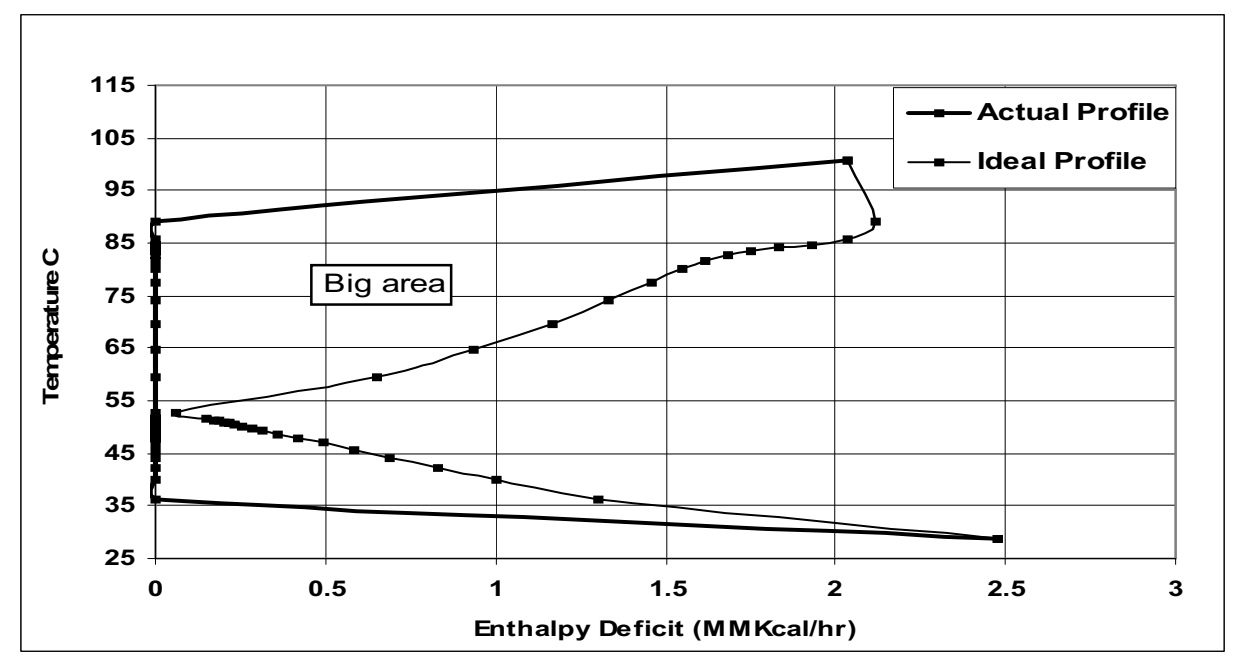

Figure 4. Debutanizer Column Grand Composite Curve after feed pre-cooling

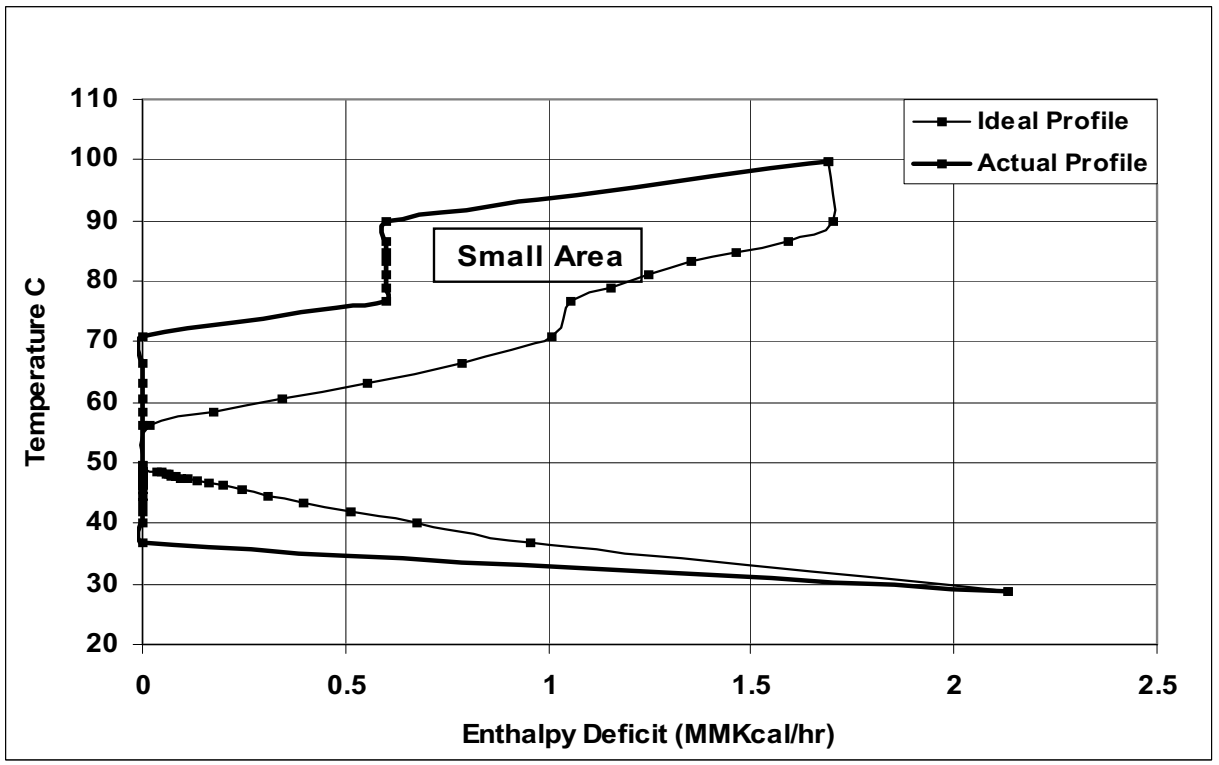

Figure 5. Debutanizer Column Grand Composite Curve after side-reboiling 


\section{Side condensing or side reboiling}

The significant area above the pinch as shown in Figure 4, dictates the need for a side reboiler, which accordingly was added to the column at stage 26, as shown in Figure 5.

\section{Conclusion}

A hierarchical approach has been introduced to optimize fractionation systems for LNG facilities. Comparing condenser and reboiler duties after applying the column targeting approach with those before, it is obvious that there was a big difference in the heat duty values. The condenser duty decreased from $3.98 \mathrm{MMKcal} / \mathrm{hr}$ to $2.13 \mathrm{MMKcal} / \mathrm{hr}$ after applying column targeting with a pre-cooler that has a heat duty of $0.44 \mathrm{MMKcal} / \mathrm{hr}$. The reboiler on the other hand decreased also from heat duty of $3.46 \mathrm{MMKcal} / \mathrm{hr}$ to 1.09 MMKcal $/ \mathrm{hr}$ after column targeting with a side reboiler of $0.6 \mathrm{MMKcal} / \mathrm{hr}$. Figure 6 shows the column design after applying the four optimization steps.

It is worth mentioning that this process is a trade off between capital and operating cost, therefore, such saving should be incorporated into the overall system cost after adding the new equipment cost, like pre-cooler, side reboiler and the addition of the new stages.

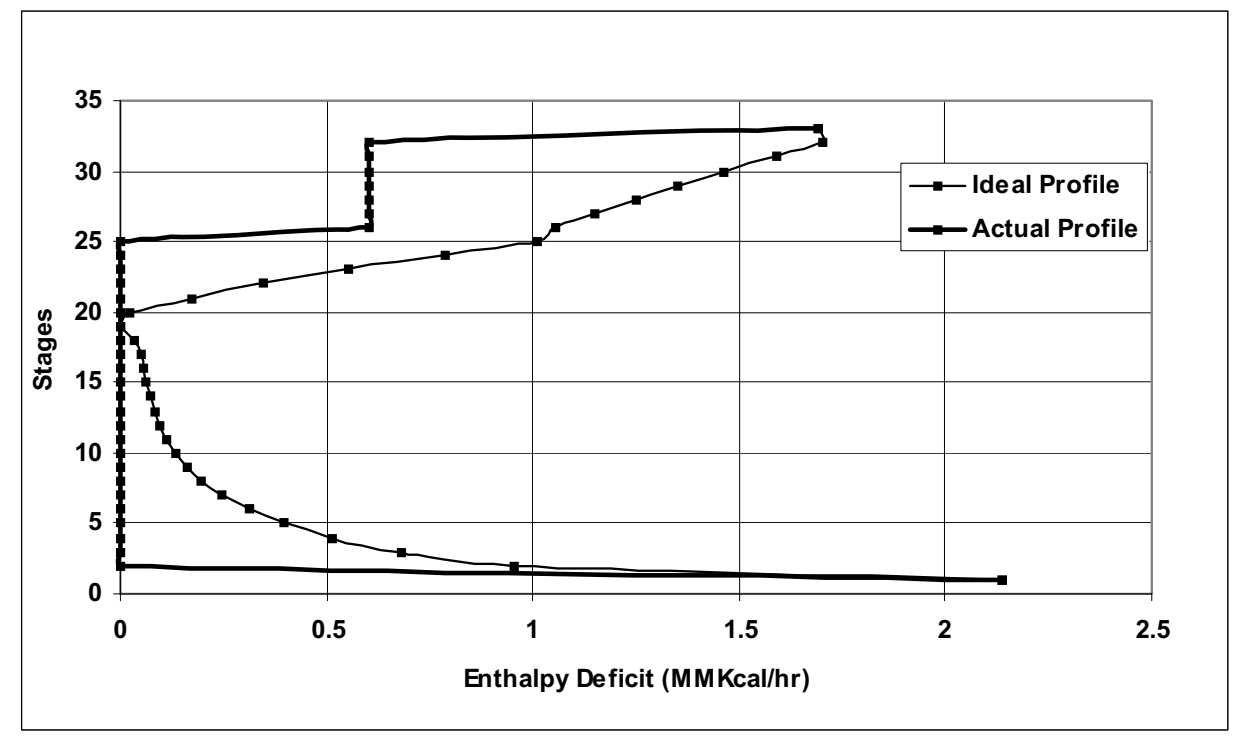

Figure 6. Debutanizer Final Design (Stage- Enthalpy) Column Grand Composite Curve

\section{References}

Aspen Technology Inc., 2003, Aspen Plus 12.1 Unit Operations Models Reference Manual, Cambridge, USA.

Dhole, V.R. and B. Linnhoff, 1993, Distillation Column Targets, Computers Chem. Engng, 17, 549-560.

Gadalla M, Jobson M, and Smith R, Optimization of heat-integrated refinery distillation systems, Trans IChemE, January, 81(A) 147-152 (2003)

Seader, J.D. and E.J. Henly, 1998, Separation Process Principles, John Wiley \& Sons, Inc. New York. 